\title{
Safety and Efficacy of Pilocarpine, Cevimeline and Diquafosol Compared to Artificial Tears for the Treatment of Dry Eye: Protocol for a Systematic Review.
}

\section{José Gerardo Serrano-Robles}

Universidad Anahuac Mexico https://orcid.org/0000-0002-2926-219X

Ana Karen Pérez-Vázquez

Universidad Anahuac Mexico

Alejandro Navas-Pérez

Instituto de Oftalmologia Fundacion Conde de Valenciana IAP

Enrique 0 Graue-Hernandez

Instituto de Oftalmologia Fundacion Conde de Valenciana IAP

Arturo Ramirez-Miranda

Instituto de Oftalmologia Fundacion Conde de Valenciana IAP

Nicolás Kahuam-López ( $\square$ nkahuam@gmail.com )

Universidad Anáhuac México and Instituto de Oftalmología Fundación Conde de Valenciana https://orcid.org/0000-0003-3887-0629

\section{Protocol}

Keywords: Systematic Review, Meta-analysis, Protocol, Secretagogues, Artificial Tears, Dry eye

Posted Date: December 28th, 2020

DOI: https://doi.org/10.21203/rs.3.rs-133476/v1

License: (c) (i) This work is licensed under a Creative Commons Attribution 4.0 International License. Read Full License 


\section{Abstract}

Background: Dry eye disease (DED) is a condition that affects the ocular surface and affects millions of people around the world. In recent years a stepped scheme has been proposed for the treatment of DED, with the use of an artificial tear being the mainstay of treatment. In this scheme, the use of secretagogues is suggested as part of the treatment for patients with moderate to severe affectation. With this systematic review we aim to evaluate the effectiveness and safety of secretagogues for DED.

Methods: Electronic databases will be searched; we will include randomized controlled trials that compare secretagogues and artificial tears. Study inclusion will not be restricted on the basis of language or publication status. We will use Google Translate to assess studies written in languages other than English and Spanish. Identification, evaluation, data extraction and assessment of risk of bias will be conducted by two authors of the review, a third review author will resolve any disagreement. The outcomes will be the ocular surface disease index score, osmolarity and tear film break-up time. We will use the Cochrane Collaboration Risk of Bias (RoB) tool for assessing the risk of bias of the included studies.

Based on the heterogeneity of the included studies, we will combine the findings in a meta-analysis using a random versus a fixed effect model. If we deem meta-analysis as inappropriate, we will document the reasons and report findings from the individual studies narratively.

Discussion: Based on the evidence obtained we will evaluate the effect of Pilocarpine, Cevimeline and Diquafosol and compare it to artificial tears on multiple outcome measures.

This systematic review aims to determine the efficacy and safety of the secretagogues pilocarpine, cevimeline and diquafosol to help clinicians in the decision-making process.

Registration: PROSPERO CRD42020218407

\section{Background}

\section{Description of The Condition}

Dry eye disease (DED) is one of the main reasons for consultation with ophthalmologists in the clinical setting, it frequently presents with foreign body sensation, burning and pain associated with blurred vision that negatively impacts the quality of life of patients. It is estimated that the prevalence ranges from $5 \%$ to $35 \%$ with a predominance of females and with a maximum peak at age 60 where the prevalence reaches $70 \%$ with a greater trend for the Asian population $(1-5)$.

The three main components of tear film are the mucin layer, the aqueous layer, and the lipid layer. Different conditions that affect one or more components of the tear film or the glands that produce its components has the potential to produce the disease(4). Tear hyperosmolarity is considered to be the 
trigger for a cascade of signaling events within corneal epithelial cells, leading to the release of inflammatory mediators and proteases (6).

The Ocular Surface Disease Index (OSDI) consists of 12 questions and its objectives are to make the diagnosis of ocular surface disease easier, faster and more reliable, as well as to provide evidence of differences in ocular disability due to $\operatorname{DED}(7,8)$. The time from the ascending trace of the last blink to the breaking of the tear film or the formation of a dry spot is recorded as the tear break-up time (TBUT) measurement. $(7,9,10)$. The osmolarity of the tear film indicates the balance of inputs and outputs of the lacrimal system. The cut-off points for making the diagnosis of DED by osmolarity is $\geq 308 \mathrm{mOsm} / \mathrm{L}$ in one or both eyes or a gradient $\geq 8 \mathrm{mOsm} / \mathrm{L}$ in the osmolarity between both eyes $(11,12)$.

\section{Description of The Intervention}

The management of DED is complicated, due to its multifactorial etiology. The goal of treating DED is to restore homeostasis to the ocular surface and tear film, breaking the vicious cycle of the disease.

Management algorithms are often constructed to recommend a sequence of treatments according to the stage of the disease, but this construction is complicated in DED, as the disease often varies from patient to patient, both in severity and in character. In recent years a scheme has been proposed for the treatment of DED (13), with the use of artificial tears being the mainstay of treatment. In this scheme, the use of secretagogues is suggested as part of the treatment for patients with moderate to severe degrees of the disease.

Management approaches begin with, low-risk, and easily accessible patient-applied therapies, such as artificial tears for early-stage disease, and progress to more advanced therapies for more severe forms of DED (14).

Various pharmacological agents with a secretagogue effect can stimulate watery secretion, mucus secretion, or both. Topical Diquafosol eye drops have been favorably evaluated in several clinical trials $(5,15,16)$. This agent is able to stimulate watery and mucous secretion in both animals and humans. It is also possible to administer orally cholinergic agonists, particularly Pilocarpine and Cevimeline, to treat severe DED. They have FDA-approved indications for the treatment of dry mouth associated with Sjögren's syndrome $(17,18)$.

\section{How the Intervention Might Work}

Secretagogue therapy suggest advantages over using artificial tears. Diquafasol solution lowers the corneal fluorescein and rose Bengal scores compared to artificial tears (19), and improves the tear break time (20). Another report suggested the superior efficacy and safety of $3 \%$ Diquafosol ophthalmic solution compared to the use of other secretagogue therapies such as Cevimeline and Pilocarpine $(18,21)$. 
Regarding the long-term efficacy and safety of $3 \%$ Diquafosol ophthalmic solution, the therapy was shown to significantly improve both subjective (dry eye symptom scores) and objective symptoms (eye

staining score and tear function tests) the major adverse reactions were eye discharge, eye irritation, and eye pain, nevertheless the symptoms remitted after 28 days (22).

The use of acetylcholine analogues has been studied as a possible alternative for Sjögren's syndrome, although most studies have shown more benefits for dry mouth, there is strong evidence that it can improve DED symptoms (23). A possible disadvantage of muscarinic agonists is that they are contraindicated in angle-closure glaucoma and should be used with care in patients with asthma and heart disease (23).

\section{Why It Is Important to Do This Review}

DED is a condition that affects the ocular surface and affects millions of people around the world, the presentation of symptoms is very varied since it ranges from a foreign body sensation to severe pain $(1,4)$, which can make activities of daily living impossible. Buchholz et al. found that patients with severe DED, dialysis, and severe angina are willing to spend the same amount of time treating their illnesses (24).

It is estimated that the prevalence of DED ranges from $5 \%$ to $35 \%$ with a predominance of females and with a maximum peak at age 60 where the prevalence reaches $70 \%$ with a greater trend for the Asian population $(1-5)$.

Therefore, it is important to evaluate the current evidence to compare the use of secretagogues with the use of artificial tears in DED, to determine the better option in terms of effectiveness and safety.

\section{OBJECTIVES}

To compare the effectiveness and safety of secretagogues versus artificial tears for dry eye.

\section{RESEARCH QUESTION}

What is the effect on the Ocular Surface Disease Index of patients with dry eye treated with Pilocarpine, Cevimeline or Diquafosol compared to the use of artificial tears?

P: Patients with dry eyes.

I: Use of Secretagogues.

C: Artificial tears.

0: Ocular surface disease index.

\section{Methods}




\section{Criteria for Considering Studies for this Review}

\section{Types of Studies}

We will include randomized controlled trials that compare secretagogues and artificial tears. Study inclusion will not be restricted on the basis of language or publication status.

\section{Types of Participants}

We will include trials where the study population comprises people 18 years old or older with dry eye disease.

\section{Types of Interventions}

Treatment groups: Secretagogues, including Pilocarpine, Cevimeline and Diquafosol

Control group: Artificial Tears intervention with different types.

\section{Types of Outcome Measures}

\section{Primary Outcomes}

- Assess the efficacy and safety of patients with dry eye treated with secretagogues.

\section{Secondary Outcomes}

- Evaluate the adverse effects of Pilocarpine, Cevimeline or Diquafosol.

- Compare the Ocular Surface Disease Index score in dry eye patients treated with Pilocarpine, Cevimeline and Diquafosol versus artificial tears.

- Compare the osmolarity before and after application of Pilocarpine, Cevimeline or Diquafosol with the use of artificial tears.

- Compare the tear film Break-up time before and after application of Pilocarpine, Cevimeline or Diquafosol with the use of artificial tears.

- Compare the Schirmer test score before and after application of Pilocarpine, Cevimeline or Diquafosol with the use of artificial tears.

- Compare the effect of Pilocarpine, Cevimeline or Diquafosol with VRQoL (Vision-Related Quality of Life) in patients with dry eye.

\section{Adverse Outcomes}


We will compare the proportion of adverse outcomes between treatment groups. We will consider adverse outcomes as reported by included studies. Specific adverse outcomes of interest will include the following:

- Eye discharge.

- Eye irritation.

- Eye pruritus.

- Eye pain.

- Foreign body sensation.

- Allergic conjunctivitis.

\section{Search Methods for Identification of Studies}

\section{Electronic Searches}

The search of information will include the following electronic databases for randomized controlled trials. There will be no language or publication year restrictions.

- Cochrane Central Register of Controlled Trials (CENTRAL) (which contains the CEV Trials Register) in the Cochrane Library (latest issue)

- PubMed (1948 to present)

- Scopus (2004 to present)

- LILACS (Latin American and Caribbean Health Science Information Database (1982 to present)

- US National Institutes of Health Ongoing Trials Register ClinicalTrials.gov

- World Health Organization (WHO) International Clinical Trials Registry Platform (ICTRP)

\section{Searching Other Resources}

We will search the reference lists of eligible studies identified from the electronic searches for additional relevant trials that may not have been identified from the electronic searches.

\section{Data Collection and Analysis}

\section{Selection of Studies}

The study selection will be performed by pairs of review authors, they will assess the titles and abstracts of articles identified through the literature search and will compare these against the inclusion criteria. Each article will be assessed independently by both authors and will be classified as either 'definitely relevant', 'possibly relevant', or 'definitely not relevant'.

Covidence software will be used to manage the screening process (25). Any disagreement will be resolved by a third review author. 
Then we will obtain the full-text copies of all studies classified as 'definitely relevant' or 'possibly relevant'. Each review author will independently assess each study for inclusion and will label it as either 'include' or 'exclude'. A third review author will resolve any disagreement. We will document the reason for exclusion of each study excluded after reviewing the full report in a 'Characteristics of excluded studies' table. We will use Google Translate to assess studies written in languages other than English and Spanish. We will illustrate the study selection process in a PRISMA diagram.

\section{Data Extraction and Management}

Pairs of review authors will independently extract data from the included studies using a data extraction form (Additional File 1) adapted from the Cochrane Eyes and Vision (CEV) data extraction form (26) developed by CEV and accessed via Covidence. A third review author will resolve any disagreements.

One review author will enter data into Review Manager 5 (RevMan 5) (27), and a second review author will verify the data entered.

\section{Assessment of Risk of Bias in Included Studies}

The Cochrane Collaboration Risk of Bias (RoB) tool will be used to assess the RoB in selection (random sequence generation and allocation concealment before randomization), performance (masking of study participants and personnel), detection (masking of outcome assessors), attrition (missing data and absence of an intention-to-treat analysis), reporting (selective outcome reporting), and other potential sources of bias (28).

Two authors of the review will classify the risk of bias as either 'low', 'high', or 'unclear' (insufficient information for assessment). A third review author will resolve any disagreement between review authors.

\section{Measures of Treatment Effect}

We will calculate mean difference (MDs) with 95\% confidence intervals (Cls) for continuous measures (e.g., the Schirmer test value, TBUT) and risk ratios (RRs) with the corresponding 95\% Cls for dichotomous outcomes to estimate effects (e.g., adverse events, effective rate). We will choose a cut-off for ordinal outcomes and measurement scales to handle them as binary data or treat them as continuous data, as appropriate.

\section{Unit of Analysis Issues}

The participant will be the primary unit of analysis whenever: a) only one eye per participant is enrolled in the trial; or b) two eyes of an individual are treated as a single unit after being administered the same treatment (e.g. Pilocarpine, Cevimeline and Diquafosol). For studies that enrolled both eyes of participant and in which the eye was the unit of analysis, we will document whether the trial had a within-person design and analyzed the data appropriately.

\section{Dealing with Missing Data}


We will contact corresponding authors by email to obtain missing data or data reported unclearly in the study reports. We will allow three weeks for study authors to respond and will use the available information whenever there is no response. We will not impute missing participant data for analysis.

\section{Assessment of Heterogeneity}

We will compare the participant characteristics, study interventions, and outcomes across trials to assess for clinical and methodological heterogeneity.

We will use the $\mathrm{I}^{2}$ statistic, which estimates the proportion of variation in observed effects not due to chance, to identify inconsistency among trials; an $\mathrm{I}^{2}$ statistic value of greater than $50 \%$ (moderate heterogeneity)will represent substantial heterogeneity (28). Chi ${ }^{2}$ test statistics will be used to assess the statistical heterogeneity among estimates of effect size from the included studies.

\section{Assessment of Reporting Biases}

If 10 studies, or more, are included we will visually inspect funnel plots of the intervention effect estimates for evidence of asymmetry to identify publication bias.

An asymmetric funnel plot may suggest small study effects, which could be the result of reporting bias, heterogeneity, or differences in the methodological quality of studies. We will assess selective outcome reporting as part of the 'Risk of bias's assessment among individual studies.

\section{Data Synthesis}

If there is no substantial heterogeneity between studies $\left(I^{2}<50 \%\right)$ we will apply the fixed effects model to synthesis the data and a random effects models if we encounter substantial heterogeneity. If we deem a meta-analysis as inappropriate, we will document the reasons and report findings from the individual studies narratively.

\section{Subgroup Analysis and Investigation of Heterogeneity}

If sufficient data are available from included studies, we will examine findings by the degree of DED severity at baseline among the study participants.

\section{Sensitivity Analysis}

Where possible, we will perform sensitivity analyses for primary and secondary outcomes to explore the effects of restricting our analyses to trials judged to have adequate allocation concealment, adequate masking of outcome assessors, and had at least $80 \%$ follow-up of participants in each group, to determine the robustness of the conclusion.

\section{Summary of findings}


When sufficient evidence is available, we will summarize the findings of the review using the Grades of Recommendation, Assessment, Development and Evaluation (GRADE) approach to assess the strengths and limitations of evidence for both primary and secondary outcomes.

\section{Discussion}

DED presents with a broad spectrum of presentation that ranges from eye discomfort to ocular pain with blurry vision, affecting quality of life and limiting activities of daily living.

Randomized controlled clinical trials have been published suggesting the use of secretagogues such as Pilocarpine, Cevimeline and Diquafosol for the treatment of this disease. This systematic review aims to determine the efficacy and safety of the secretagogues pilocarpine, cevimeline and diquafosol to help clinicians in the decision-making process.

\section{List Of Abbreviations}

DED, Dry eye disease; OSDI, Ocular Surface Disease Index; RoB, Risk of Bias; TBUT, Tear Break-up time; VRQoL, Vision-Related Quality of Life; RevMan 5, Review Manager 5; CEV, Cochrane Eyes and Vision; MDs, mean difference; Cls, Confidence Intervals; RRs, Risk Ratios;

\section{Declarations}

\section{Ethics approval and consent to participate}

'Not applicable'

Consent for publication

'Not applicable'

\section{Availability of data and materials}

'Not applicable'

\section{Competing interests}

'Not applicable'

\section{Funding}

'Not applicable'

\section{Authors' contributions}

All review authors have contributed to the production of the protocol. 
All authors read and approved the final manuscript. JGSR and NKL led the writing of the protocol, AKPV, ANP, EOGH and ARM provided comments and feedback.

\section{Acknowledgements}

We would like to thank the Center for Research in Health Sciences (CICSA), Faculty of Health Sciences, Universidad Anáhuac México, Campus Norte, for providing the necessary databases to develop the search strategy, we also thank the Cornea and Refractive Surgery Department, Instituto de Oftalmología Fundación Conde de Valenciana, for providing recommendations and feedback.

\section{References}

1. Schaumberg DA, Nichols JJ, Papas EB, Tong L, Uchino M, Nichols KK. The international workshop on meibomian gland dysfunction: Report of the subcommittee on the epidemiology of, and associated risk factors for, MGD. Investig Ophthalmol Vis Sci. 2011;52(4):1994-2005.

2. Nichols KK. The international workshop on meibomian gland dysfunction: Introduction. Investig Ophthalmol Vis Sci. 2011;52(4):1917-21.

3. Daniel Nelson J, Shimazaki J, Benitez-del-Castillo JM, Craig J, McCulley JP, Den S, et al. The international workshop on meibomian gland dysfunction: Report of the definition and classification subcommittee. Investig Ophthalmol Vis Sci. 2011;52(4):1930-7.

4. Clayton JA. Dry eye. N Engl J Med. 2018;378(23):2212-23.

5. Dana R, Bradley JL, Guerin A, Pivneva I, Stillman IÖ, Evans AM, et al. Estimated Prevalence and Incidence of Dry Eye Disease Based on Coding Analysis of a Large, All-age United States Health Care System. Am J Ophthalmol. 2019;202:47-54.

6. Knop E, Knop N, Millar T, Obata H, Sullivan DA. The international workshop on meibomian gland dysfunction: Report of the subcommittee on anatomy, physiology, and pathophysiology of the meibomian gland. Investig Ophthalmol Vis Sci. 2011;52(4):1938-78.

7. Miller KL, Walt JG, Mink DR, Satram-Hoang S, Wilson SE, Perry HD, et al. Minimal clinically important difference for the ocular surface disease index. Arch Ophthalmol. 2010;128(1):94-101.

8. Uchino M, Schaumberg DA. Dry Eye Disease: Impact on Quality of Life and Vision. Curr Ophthalmol Rep. 2013;1(2):51-7.

9. Green-Church KB, Butovich I, Willcox M, Borchman D, Paulsen F, Barabino S, et al. The international workshop on meibomian gland dysfunction: Report of the subcommittee on tear film lipids and lipidprotein interactions in health and disease. Investig Ophthalmol Vis Sci. 2011;52(4):1979-93.

10. Tomlinson A, Bron AJ, Korb DR, Amano S, Paugh JR, lan Pearce E, et al. The international workshop on meibomian gland dysfunction: Report of the diagnosis subcommittee. Investig Ophthalmol Vis Sci. 2011;52(4):2006-49.

11. Craig JP, Nelson JD, Azar DT, Belmonte C, Bron AJ, Chauhan SK, et al. TFOS DEWS II Report Executive Summary. Ocul Surf. 2017;15(4):802-12. 
12. Wolffsohn JS, Arita R, Chalmers R, Djalilian A, Dogru M, Dumbleton K, et al. TFOS DEWS II Diagnostic Methodology report. Ocul Surf [Internet]. 2017;15(3):539-74. Available from: http://dx.doi.org/10.1016/j.jtos.2017.05.001

13. Rauz S, Koay S-Y, Foot B, Kaye SB, Figueiredo F, Burdon MA, et al. The Royal College of Ophthalmologists guidelines on serum eye drops for the treatment of severe ocular surface disease: full report. Eye. 2017;1-16.

14. Jones L, Downie LE, Korb D, Benitez-del-Castillo JM, Dana R, Deng SX, et al. TFOS DEWS II Management and Therapy Report. Ocul Surf [Internet]. 2017;15(3):575-628. Available from: http://dx.doi.org/10.1016/j.jtos.2017.05.006

15. Park CH, Lee HK, Kim MK, Kim EC, Kim JY, Kim TI, et al. Comparison of $0.05 \%$ cyclosporine and $3 \%$ diquafosol solution for dry eye patients: A randomized, blinded, multicenter clinical trial. BMC Ophthalmol. 2019;19(1):1-10.

16. Shimazaki J, Seika D, Saga M, Fukagawa K, Sakata M, Iwasaki M, et al. A Prospective, Randomized Trial of Two Mucin Secretogogues for the Treatment of Dry Eye Syndrome in Office Workers. Sci Rep. 2017;7(1):1-10.

17. Ono M, Takamura E, Shinozaki K, Tsumura T, Hamano T, Yagi Y, et al. Therapeutic effect of cevimeline on dry eye in patients with Sjögren's syndrome: A randomized, double-blind clinical study. Am J Ophthalmol. 2004;138(1):6-17.

18. Bohm KJ, Djalilian AR, Pflugfelder SC, Starr CE. Part v The Ocular Adnexa Section 4 Disorders of Tear Production and the Lacrimal System Dry Eye Lacrimal Functional Unit: Anatomy and Physiology. Cornea. 2017;377-95.

19. Takamura E, Tsubota K, Watanabe H, Ohashi Y. A randomised, double-masked comparison study of diquafosol versus sodium hyaluronate ophthalmic solutions in dry eye patients. $\mathrm{Br} \mathrm{J}$ Ophthalmol. 2012;96(10):1310-5.

20. Fukuoka S, Arita R. Tear film lipid layer increase after diquafosol instillation in dry eye patients with meibomian gland dysfunction: a randomized clinical study. Sci Rep. 2019;9(1):1-11.

21. Gong L, Sun X, Ma Z, Wang Q, Xu X, Chen X, et al. A randomised, parallel-group comparison study of diquafosol ophthalmic solution in patients with dry eye in China and Singapore. Br J Ophthalmol. 2015;99(7):903-8.

22. Watanabe H. Medical treatment for dry eye in Japan. Investig Ophthalmol Vis Sci. 2018;59(14 Special Issue):DES116-20.

23. Valim V, Trevisani VFM, de Sousa JM, Vilela VS, Belfort R. Current Approach to Dry Eye Disease. Clin Rev Allergy Immunol. 2015;49(3):288-97.

24. Buchholz P, Steeds CS, Stern LS, Wiederkehr DP, Doyle JJ, Katz LM, et al. Utility assessment to measure the impact of dry eye disease. Ocul Surf. 2006;4(3):155-61.

25. Veritas Health Innovation. Covidence. [Internet]. Melbourne, Australia: Veritas Health Innovation, 2020; [cited 2020 Sep 5]. Available from: https://www.covidence.org/

26. Navas A, Eo G, Ibarra A. LASIK in adults with myopia or myopic astigmatism ( Review ). 2020; 
27. Collaboration TC. Review Manager 5 (RevMan 5). Copenhagen: Nordic Cochrane Centre; 2014. p. 143.

28. Higgins JP, Green S E. Cochrane Handbook for Systematic Reviews of Interventions Version 5.1.0. Cochrane Collab [Internet]. 2011;(March):1-639. Available from: www.cochrane-handbook.org

\section{Appendix}

Appendix 1: search strategy.

\section{PubMed}

\#1 ((randomized controlled trial[pt]) OR (controlled clinical trial[pt]) OR (randomized[tiab] OR randomized[tiab]) OR (randomly[tiab]) OR (trial[tiab]) OR (groups[tiab])) NOT (animals[mh] NOT humans[mh])

\#2 (Dry eye [tw] OR Dry eye syndrome [tw] OR Dry eye disease [tw] OR Conjunctivitis sicca [tw] OR Keratoconjunctivitis sicca [tw] OR Keratitis sicca [tw])

\#3 (Pilocarpine [pa] OR Cevimeline [pa] OR Diquafosol [pa]) OR (Pilocarpine [tiab] OR Cevimeline [tiab] OR Diquafosol [tiab])

\#4 (Artificial tear [tiab] OR Ocular lubricant [tiab]) OR (Artificial tear [pa] OR Ocular lubricant [pa])

\#5 \#1 AND \#2 OR \#3 OR \#OR

Cochrane Central Register of Controlled Trials (CENTRAL)

\#1 MeSH descriptor: [Dry Eye] explode all trees

\#2 MeSH descriptor: [Dry Eye Disease] explode all trees

\#3 MeSH descriptor: [Dry Eye Syndrome] explode all trees

\#4 (Pilocarpine):kw

\#5 (Cevimeline):kw

\#6 (Diquafosol):kw

\#7 (Artificial tear):kw

\#8 (Ocular lubricant):kw

\#9 \#1 OR \#2 OR \#3 OR \#4 OR \#5 \#6 OR \#7 OR \#8

\section{LILACS}

Page 12/13 
(TW:"randomized controlled trial" OR TW:"controlled clinical trial" OR TW:randomized OR TW:randomized OR TW:randomly OR TW:trial OR TW:groups) AND ((TW:"Dry eye" OR TW:"Dry eye syndrome" OR TW:"Dry eye disease" OR TW:"Conjunctivitis sicca" OR TW:"Keratoconjunctivitis sicca" OR TW:"Keratitis sicca") OR (TW:Pilocarpine OR TW:Cevimeline OR TW:Diquafosol) OR (TW:"Artificial tear" OR TW:"Ocular lubricant"))

\section{ClinicalTrials.gov}

((Dry eye OR Dry eye syndrome OR Dry eye disease OR Conjunctivitis sicca OR Keratoconjunctivitis sicca OR Keratitis sicca) OR (Pilocarpine OR Cevimeline OR Diquafosol) OR (Artificial tear OR Ocular lubricant))

\section{WHO ICTRP}

((Dry eye OR Dry eye syndrome OR Dry eye disease OR Conjunctivitis sicca OR Keratoconjunctivitis sicca OR Keratitis sicca) OR (Pilocarpine OR Cevimeline OR Diquafosol) OR (Artificial tear OR Ocular lubricant))

\section{Scopus}

( ( randomized AND controlled AND trial) OR ( controlled AND clinical AND trial ) OR ( randomized OR randomized) OR ( randomly) OR ( trial) OR ( groups ) ) AND (dry AND eye OR dry AND eye AND syndrome OR dry AND eye AND disease OR conjunctivitis AND sicca OR keratoconjunctivitis AND sicca OR keratitis AND sicca ) AND ( pilocarpine OR cevimeline OR diquafosol ) OR ( artificial AND tear OR ocular AND lubricant )

\section{Supplementary Files}

This is a list of supplementary files associated with this preprint. Click to download.

- DataextractionformAdditionalFile1.docx

- PRISMAPchecklist.pdf 